\title{
DIÁLOGO DE SABERES: MEDICINA TRADICIONAL Y MEDICINA OCCIDENTAL MODERNA
}

\author{
Maristela Jácome Romero y \\ Erika Bayona Rodríguez ${ }^{11}$
}

\begin{abstract}
RESUMEN
En este espacio se adelantarán algunas reflexiones basadas en el avance del proyecto Estado del Arte de la Etnomedicina en Colombia, con el propósito de dar a conocer una de las categorías más representativas alrededor del tema como lo es "El diálogo de saberes" entre las medicinas tradicionales y la medicina moderna occidental desde el marco de la interculturalidad como una de las características más representativas de las Ciencias Sociales y Educativas. "El diálogo de saberes" se plantea como una tendencia dentro de los estudios culturales que establece un diálogo entre la academia, los investigadores, las instituciones en este caso de Salud Pública y los conocimientos de las comunidades a partir de la Investigación Acción Participativa, Las Sinergias Colectivas y el empoderamiento de las comunidades con el propósito de producir nuevos conocimientos y dinámicas de participación desde ambas realidades.
\end{abstract}

Históricamente, las relaciones entre ambas culturas han sido traumáticas. Con las minorías étnicas han predominado la inequidad, la discriminación y la exclusión por parte de las políticas y el desconocimiento de la sociedad mayoritaria que solo ha tenido acercamientos a los saberes tradicionales con un propósito mercantilista o utilitario. Ante este panorama, se presentan aquí los intentos por generar nuevas propuestas de acercamiento más adecuadas, donde el diálogo intercultural sea la base para fomentar el reconocimiento entre estos Sistemas, dando cumplimiento a la Línea de Investigación que enmarca el presente proyecto: La Visibilidad y la Gestión de Conocimiento.

Por ser un Estado del Arte, la metodología ha sido exploratoria y descriptiva a partir de la búsqueda de publicaciones encontradas en bases de datos físicas y electrónicas, para luego establecer las categorías de análisis y los enfoques. Se abordará esta categoría desde los conceptos de salud y enfermedad y las principales características de cada uno, las dinámicas interculturales que se están generando, indagando el presente y el futuro de este diálogo desde los escenarios y actores, tomando como referencia la principal herramienta: la Etno-medicina, como disciplina emergente y su marco de acción.

Palabras clave: diálogo de saberes, Etno-medicina, medicina tradicional, medicina occidental, comunidad, interculturalidad.

11 Grupo de Investigación: Gestión Vital, Código: COL0056645, Semillero: Júpiter, Línea de Investigación: Visibilidad y Gestión de Conocimiento, Red de Investigación: Visibilidad y Gestión de Conocimiento. E-mail: erika.bayona@live.com. 


\begin{abstract}
In this space they will be brought forward some reflections based on the progress of the project State of the Art of Ethnomedicine in Colombia, in order to make known one of the most representative categories about "Dialogue of Knowledge" between traditional medicines and modern Western medicine from the framework of Interculturalism as one of the most representative characteristics of the Social Sciences and Education. The Dialogue of knowledge is seen as a tendency within cultural studies that establishes a dialogue among Academy, Researchers, and Institutions of Public Health in this case, the expertise of communities from Participatory Action Research, The Collective Synergy and empowerment of communities in order to produce new knowledge and dynamics of participation from both realities.
\end{abstract}

Historically, relations between the two cultures have been traumatic for the inequality, discrimination, exclusion to what ethnic minorities have been subjected by the policies and the lack of mainstream society, in which approaches to traditional knowledge have mercantilist or utilitarian purpose. In that sense, they are attempts to generate new proposals for more appropriate approaches where intercultural dialogue is the basis for promoting the recognition between these systems and therefore comply with the research line that frames this project, The Visibility and Management Knowledge.

As it is a State of the Art, the methodology has been exploratory and descriptive based on the search of publications found on physical and electronic databases, and publications of communities, and then to establish the categories of analysis and approaches.

This category will be approached from the concepts of health and disease and the principal characteristics of each one, the intercultural dynamics that are generated, investigating the present and the future of this one dialog from the scenes and actors, taking as a reference the principal tool the Etnomedicina, as emergent discipline and his frame of action

Key words: dialogue of Knowledge, ethno-medicine, traditional medicine, Western medicine, community, intercultural

\title{
INTRODUCCIÓN
}

Desde que la Organización Mundial de la Salud (OMS) incorpora en sus programas el interés por el estudio de las Medicinas Tradicionales en 1975, son muchas las acciones que se vienen adelantando con relación a las políticas y a los procesos interculturales entre ambos saberes; muestra de ello es el interés de los médicos por conocer las prácticas tradicionales en salud de las comunidades y el interés de los practicantes de medicina tradicional por acercarse y reconocer las característica y prácticas de la medicina occidental.

Desde hace décadas, y como principal aporte de la antropología simbólica y médica, se ha considerado la visión de las comunidades minoritarias como depositaria de prácticas, uso de 
plantas y tratamientos que pueden aportar a los conocimientos de las Ciencias de la Salud. Sin embargo, esta mirada lejos de beneficiar a las comunidades ha traído graves consecuencias ante la apropiación y usurpación del conocimiento a que han sido sometidas. Dicha situación está modificando, aunque lentamente, la legislación de los Organismos Internacionales que acogen y protegen la integridad de las minorías étnicas, como también de las legislaciones nacionales donde hay presencia de Grupos Étnicos.

Uno de los problemas más serios es la comercialización de los recursos del planeta desde la mirada mercantil de Occidente donde, como dice (Scott, 1998), citado por Dover: "el discurso utilitario reemplaza el término naturaleza con el término de recursos naturales, enfocando en los aspectos de la naturaleza que pueden ser apropiados para el uso humano las matas que tengan valor llegan a ser cultivos, las especies que compiten con ellas hierbas malas, los árboles que tengan valor llegan a ser árboles maderables”. Además, Aguirre (2000) citando a Agrawal (2002), cuando sostiene que: "solo el saber útil se convierte en algo digno de protección”, es quien plantea la idea de protección de estos conocimientos generando paralelamente alternativas de acercamiento.

Desde las Ciencias Sociales y Educativas existe un diálogo intercultural que pone en marcha lo consignado en la Constitución Nacional de 1991 donde se hace el reconocimiento del país como pluriétnico y multicultural y desde las pedagogías del Constructivismo y la Teoría Crítica que empoderan los discursos y las prácticas locales de las minorías.

En el estudio se destacan las investigaciones de la Universidad Nacional de Colombia y, principalmente, los adelantos conceptuales en la Etnomedicina del doctor Germán Zuluaga de la Universidad del Rosario, así como las prácticas con varias comunidades del Pie de monte amazónico.

\section{OBJETIVO GENERAL}

Identificar los conceptos, antecedentes y dinámicas interculturales que se desarrollan con relación a las Medicinas Tradicionales y Occidentales a partir del Diálogo de Saberes.

\section{OBJETIVO ESPECÍFICOS}

Identificar los antecedentes que dieron origen al intercambio de saberes

Reconocer y diferenciar las principales características de los Sistemas Médicos existentes en los conceptos de salud y de enfermedad

Acercar las nociones del diálogo intercultural y los principales avances con relación al tema 


\section{METODOLOGÍA}

\section{Tipo de Estudio}

El tipo de estudio es exploratorio y descriptivo propio de un Estado de Conocimiento, en el que a partir de la identificación de categorías y el posterior análisis, se revisan las tendencias y las perspectivas con relación al tema. Se ubican y analizan todas las publicaciones referentes a este asunto a partir de las palabras clave: etnomedicina, sanación, diálogo de saberes, medicinas tradicionales, entre otras.

La población objeto de estudio son las comunidades étnicas y las instituciones académicas que estén abordando el tema principalmente desde la antropología, medicina, pedagogía, biología.

\section{Métodos}

Primero se hizo una búsqueda de artículos en bases de datos electrónicas y físicas, posteriormente se desarrolló el registro de la información en documentos RAE (Resumen Analítico Especializado) y en Mapas Conceptuales que permiten la síntesis de los artículos.

A continuación, se establecieron las categorías de búsqueda con base en la información encontrada en las publicaciones, identificando los enfoques y tendencias encontradas desde diferentes posiciones y actores.

Finalmente, se realizó la matriz incorporando los datos identificados con relación a las categorías encontradas, los artículos, autores y la descripción de los conceptos analizados, aplicando subcategorías si se requieren. Luego se realiza un escrito que presente y explique los enfoques encontrados y su relación con los demás elementos y variables para ir dando respuesta al fenómeno estudiado.

A continuación se presenta un ejemplo de la organización de las categorías de análisis, en este caso del "Diálogo de Saberes". 
Tabla 1. Ejemplo análisis de categoría y cuadro de relaciones

\begin{tabular}{|c|c|c|c|c|}
\hline Categoría & Autores & Artículos & Descripción & Subcategorías \\
\hline Diálogo de Saberes & Germán Zuluaga & $\begin{array}{l}\text { Compilación Seminario } \\
\text { Internacional } \\
\text { de Etnomedicina } \\
\text { Medicinas Tradicionales: } \\
\text { Introducción al estudio de } \\
\text { los sistemas tradicionales } \\
\text { de salud y su relación con } \\
\text { la medicina moderna. }\end{array}$ & $\begin{array}{l}\begin{array}{l}\text { Mostró los resultados } \\
\text { del primer seminario in- } \\
\text { ternacional de Etnome- } \\
\text { dicina a partir de: }\end{array} \\
\begin{array}{l}\text { Las investigaciones y } \\
\text { trabajos aplicados }\end{array} \\
\begin{array}{l}\text { Las experiencias y los } \\
\text { programas de articulación }\end{array} \\
\text { Los abordajes Académicos. } \\
\text { El marco jurídico y los } \\
\text { servicios de salud } \\
\text { Las reflexiones y los } \\
\text { marcos conceptuales. }\end{array}$ & $\begin{array}{l}\text { Niveles de Articulación } \\
\text { Estudios desde el enfo- } \\
\text { que de la medicina tra- } \\
\text { dicional } \\
\text { Estudios desde el en- } \\
\text { foque de la medicina } \\
\text { occidental. } \\
\text { Estudios desde el enfo- } \\
\text { que pedagógico. } \\
\text { Equilibrio en la concep- } \\
\text { ción y práctica de los dos } \\
\text { sistemas médicos } \\
\text { Legislación: Actualiza- } \\
\text { ción del marco legal. } \\
\text { Estrategias de articu- } \\
\text { lación entre Medicina } \\
\text { Tradicional y Medicina } \\
\text { Moderna. }\end{array}$ \\
\hline & Giovanna Reyes & $\begin{array}{l}\text { Salud Sexual y Repro- } \\
\text { ductiva bajo un nuevo } \\
\text { concepto: Reproducción } \\
\text { social }\end{array}$ & $\begin{array}{l}\text { Se hace un análisis de la } \\
\text { construcción del cono- } \\
\text { cimiento y las prácticas } \\
\text { de la comunidad indíge- } \\
\text { na en contraste con los } \\
\text { programas de cuidado } \\
\text { que se ofrecen para ellos, } \\
\text { abriendo así el campo } \\
\text { metodológico de la salud } \\
\text { pública hacia la construc- } \\
\text { ción de acciones para la } \\
\text { promoción de la salud y } \\
\text { prevención de la enfer- } \\
\text { medad, basada en la di- } \\
\text { námica del grupo social. }\end{array}$ & Salud Pública \\
\hline & Camilo Correal & $\begin{array}{l}\text { Medicinas Tradicionales } \\
\text { Introducción a la estu- } \\
\text { dio de los sistemas tra- } \\
\text { dicionales de salud y su } \\
\text { relación con la medicina } \\
\text { moderna. } \\
\text { Salud Pública y Medici- } \\
\text { na Tradicional }\end{array}$ & $\begin{array}{l}\text { Se hace la precisión so- } \\
\text { bre el significado que } \\
\text { aquí tienen las estructu- } \\
\text { ras médicas, entendiendo } \\
\text { pues, la medicina comu- } \\
\text { nitaria como la estrategia } \\
\text { que contribuye a proyec- } \\
\text { tar la influencia de las } \\
\text { acciones de los servicios } \\
\text { médicos a la comunidad, } \\
\text { integrando las activida- } \\
\text { des preventivas con las } \\
\text { asistenciales. }\end{array}$ & $\begin{array}{l}\text { Salud comunitaria } \\
\text { Como aquella en la que } \\
\text { hacen parte otras discipli- } \\
\text { nas y factores tales como } \\
\text { el contexto socio-econó- } \\
\text { mico, el medio ambiente, } \\
\text { la dinámica cultural desde } \\
\text { las dimensiones comuni- } \\
\text { cativa, epidemiológica, } \\
\text { ética y política, entre } \\
\text { otras. }\end{array}$ \\
\hline
\end{tabular}




\begin{tabular}{|c|c|c|c|c|}
\hline & $\begin{array}{l}\text { Edgar Eduardo } \\
\text { Bolívar }\end{array}$ & $\begin{array}{l}\text { Medicinas y Encuentros } \\
\text { Heterogéneos: Una mira- } \\
\text { da hacia el conflicto y el } \\
\text { diálogo de saberes }\end{array}$ & $\begin{array}{l}\text { Análisis alrededor de los } \\
\text { esfuerzos de articulación } \\
\text { entre la medicina tradi- } \\
\text { cional y la salud pública } \\
\text { que enfrenta numerosos } \\
\text { desafíos porque se tras- } \\
\text { luce más el proceso de } \\
\text { validación y traducción } \\
\text { científica de las medici- } \\
\text { nas tradicionales que el } \\
\text { desarrollo de éstas por } \\
\text { incorporar y adoptar en } \\
\text { éstas los conceptos de } \\
\text { salud y de enfermedad } \\
\text { "no modernos" }\end{array}$ & $\begin{array}{l}\text { Medicina Tradicional } \\
\text { Conjunto de conoci- } \\
\text { mientos y prácticas que } \\
\text { tienen como fundamento } \\
\text { el saber médico ancestral } \\
\text { de la población, modi- } \\
\text { ficado a lo largo de los } \\
\text { siglos por diferentes } \\
\text { factores. Se transmite } \\
\text { por tradición familiar o } \\
\text { comunitaria. }\end{array}$ \\
\hline & $\begin{array}{l}\text { Martha Cecilia } \\
\text { Suarez, Mauricio } \\
\text { Díaz }\end{array}$ & $\begin{array}{l}\text { Mujer y Salud: propuesta } \\
\text { para educación en salud } \\
\text { con mujeres indígenas } \\
\text { del Amazonas. }\end{array}$ & $\begin{array}{l}\text { Se presentan los resulta- } \\
\text { dos de una propuesta de } \\
\text { educación en salud diri- } \\
\text { gido a y construido con } \\
\text { mujeres indígenas del } \\
\text { municipio de Puerto Na- } \\
\text { riño, que se esforzó por } \\
\text { explorar nuevas maneras } \\
\text { de aprendizaje a través } \\
\text { del intercambio de cono- } \\
\text { cimientos y repensando } \\
\text { modelos pedagógicos } \\
\text { y didácticos que fueran } \\
\text { más adecuados a los } \\
\text { conceptos de salud y en- } \\
\text { fermedad de los pueblos } \\
\text { indígenas, y en especial } \\
\text { de las mujeres. }\end{array}$ & $\begin{array}{l}\text { Atención primaria en } \\
\text { salud. Como la atención } \\
\text { ambulatoria basada en } \\
\text { métodos y tecnologías } \\
\text { científicamente funda- } \\
\text { mentadas y socialmente } \\
\text { aceptables, puesta al al- } \\
\text { cance de la comunidad, } \\
\text { comprendiendo en esto } \\
\text { al individuo, la familia y } \\
\text { el ambiente, cuyo fin úl- } \\
\text { timo es la promoción de } \\
\text { la salud y prevención de } \\
\text { la enfermedad. }\end{array}$ \\
\hline
\end{tabular}

\section{RESULTADOS}

Dentro de los resultados encontramos que el estudio del Diálogo de Saberes lo encabezan el intercambio de conocimientos y las dinámicas emergentes entre la academia y las comunidades. Principalmente las primeras son las más interesadas en asumir un intercambio cultural que aporte a las Ciencias, las cuales tienen como tendencia la articulación y la observación de los saberes de las minorías tanto étnicas como culturales en varios aspectos.

De la misma forma, las comunidades en el caso del Pie de Monte Amazónico están pidiendo capacitación en programas de salud pública, en atención primaria, en salud, etc., sin embargo, los programas que se vienen aplicando desde la Secretaría de Salud del municipio no son acordes con las necesidades, los usos y costumbres de las comunidades, por lo cual éstos programas resultan inútiles y contraproducentes porque el lenguaje es totalmente occidental, al igual que los métodos de intervención. Por ésta razón, investigadores de la Universidad Nacional de la sede Amazonía han prestado atención a estas necesidades de la población, articulando los conocimientos de la medicina occidental y los conocimientos ancestrales en la atención primaria, sin atentar contra los saberes y prácticas de la población, por el contrario, fortaleciendo la identidad, las posibilidades de acción y de organización comunitaria. 
Asimismo, desde el marco conceptual se entiende que en el Diálogo de Saberes hacen parte, los actores y escenarios desde diferentes lógicas y enfoques con el propósito de desarrollar nuevos conocimientos. A partir de este punto podemos concluir que son tan diversos los investigadores y las miradas que participan del diálogo de saberes y con diferentes propósitos, que dentro del mismo enfoque se contemplan los marcos legales que protegen los saberes de ambas partes como el Código de Ética de la Medicina Indígena o las Estrategias sobre Medicina Tradicional OMS 2002-2005, entre otras.

El intercambio de saberes y prácticas, aunque viene siendo estudiado y explorado con el propósito de transformar las problemáticas y los intereses colonialistas, continua teniendo tensiones e intereses diferentes tanto constructivos como destructivos ya que esta transformación del conocimiento sigue moldeada por las formas de poder, legitimidad y validación disponibles a los diferentes actores involucrados, sin contar que la salud es un negocio con fuertes intereses económicos y político que median en estos acercamientos.

Por otro lado, el diálogo de saberes se plantea como un espacio de discusión donde se continúan construyendo los acuerdos que definen las dinámicas interculturales que se vienen estableciendo entre los dos saberes, básicamente las políticas y las económicas, en los sectores de salud - educación y población civil, los cuestionamientos del uso de las plantas medicinales y los productos farmacéuticos.

\section{DISCUSIÓN}

La discusión se centra en el abordaje de la Etnomedicina y por ende su principal enfoque: el Diálogo de saberes en el que se analizan los antecedentes más importantes que generaron este fenómeno de intercambio de conocimientos entre dos sistemas de pensamiento en este caso, desde la medicina. Dentro de esta discusión cabe anotar que los principales teóricos del tema que pertenecen a disciplinas como la Medicina y la Antropología tienen visiones opuestas en relación con la forma de abordar los conocimientos sobre el tema.

El primero lo observa desde las características de los Sistemas Médicos Occidentales sin perder las particularidades de un diálogo intercultural en el que las minorías ya no son objeto de investigación, sino que se convierten en sujeto y protagonista de la misma y éste actúa como mero observador o participante de las prácticas que allí se establezcan. El segundo afirma que la propuesta del diálogo de saberes se enmarca desde las ciencias sociales como una forma de darle sentido al universo de manifestaciones y simbolismos que se presentan entre ambos Sistemas de Conocimiento y que el solo estudio desde la Medicina Occidental, académico, no es suficiente para abordar el fenómeno.

Cierto o no, existe el creciente interés de las partes por acercarse y ampliar e integrar el conocimiento, y los participantes a éste llamado pueden surgir desde todo tipo de sectores e intereses; desde las medicinas alternativas, las medicinas occidentales, las medicinas tradicionales, turistas, simpatizantes, escritores, funcionarios públicos, entre otros. Por eso, es necesario avizorar el futuro de estas prácticas a partir de la protección, el conocimiento y el respeto por 
los saberes que surjan entre este intercambio, por lo cual emerge la disciplina denominada Etnomedicina, como aquella que reúne las investigaciones, marcos teóricos y discusiones sobre el tema.

De la misma forma, la discusión se enmarca en establecer los acuerdos alrededor de las principales problemáticas que enfrentan ambas posiciones que dificultan su interacción desde diferentes aspectos. Hay que tener en cuenta las características sociales y los escenarios participativos reales y equitativos para ir más allá de lo meramente instrumental y dominante en los ecosistemas y tratarlos como lugares de construcción de cultura, sabiduría y sociedad, de los cuales se encarga, desde los conceptos de salud y enfermedad, la nueva disciplina: Etnomedicina.

\section{CONCLUSIONES}

La existencia de dos Sistemas Médicos refleja la fortaleza para mantenerse en el tiempo a pesar de tener profundas diferencias conceptuales que, a simple vista, harían inviable una aproximación entre éstos. De ésta forma, el Diálogo de Saberes se presenta desde las características propias de cada uno de los Sistemas, encontrando puntos en común que pueden ser tomados para validar y fortalecer la experiencia de los actores y los espacios que intervienen.

La tarea inmediata consiste en consolidar un marco conceptual donde intervengan los acuerdos establecidos desde ambas posiciones a partir de los encuentros, reflexiones y estrategias de las organizaciones en las que se atiendan las necesidades, acuerdos y enfoques establecidos para generar un verdadero diálogo de saberes, acogiendo el llamado de la Intercultural como el proceso vigente para establecer los estudios sociales y comunitarios.

Desde los Sistemas Médicos surge la necesidad de abordar el universo cultural y biológico que dentro del panorama académico mundial se establece a partir de la disciplina conocida como Etnomedicina, además de ser una herramienta al servicio de la conservación de la diversidad biocultural y del mejoramiento de la salud en las comunidades. Se puede proyectar esta disciplina como un campo de acción bastante estudiado y discutido desde la interdisciplinariedad que la compone y como marco de referencia de las nuevas pedagogías y estudios interculturales futuros. Por esto la pregunta fundamental que dirige estas investigaciones es: ¿Hacia dónde se conducen las investigaciones alrededor del tema? ¿Cuál es propósito fundamental?

Terminamos con un pensamiento de uno de los investigadores que más ha aportado al marco conceptual, el doctor Germán Zuluaga: "Para que la medicina occidental sea capaz de dialogar verdaderamente con otros sistemas médicos necesita que se permita una crítica y una práctica autocrítica. No es posible dialogar con base en la igualdad cuando no se acepte ser criticado y sí criticar". 


\section{REFERENCIAS BIBLIOGRÁFICAS}

AGRAWAL,Arun(2002). Elconocimientoindígenayladimensiónpolíticadelaclasificación. En:"Revista internacional de Ciencias Sociales. N.0 173. Consejero editorial Arun Agrawal. pp. 6-18.

AGUIRRE, L. Daniel. (2009), Destilación del Conocimiento Indígena. En: las memorias de la publicación "Diálogo de Saberes: plantas medicinales, salud y cosmovisiones" Seminario de experiencias de medicina tradicional al servicio de la salud pública, realizado en Leticia entre el 10 y el 12 de mayo del 2007 por la Universidad Nacional de Colombia, sede Amazonia.

DOVER, Robert. (2004), "El encuentro inminente de otros recíprocos: estrategias para el reconocimiento de saberes plurales". Revista Educación y Pedagogía. Universidad de Antioquia-Facultad de Educación. Vol. XVI. No. 39. pp. 11-15.

GRANADOS, A, MARTÍNEZ, L., y MORALES, P.(2006),Aproximación a la Medicina Tradicional Colombiana: una mirada al margen de la cultura occidental, Revista ciencias de la salud.

GRANADOS Andrade Sandy Marcela, MARTINEZ Luis Eduardo, MORALES Paola, Aproximación a la Medicina Tradicional Colombiana: Una mirada al margen de la cultura occidental, Universidad del Rosario, Revista Ciencias de la Salud, enero-junio. vol 3, No. 001, , p.p 98 - 106, Bogotá, 2005.

GRANADOS Andrade Sandy Marcela, MARTINEZ Luis Eduardo y MORALES Paola, /2005), Aproximación a la Medicina Tradicional Colombiana: Una mirada al margen de la cultura occidental, Universidad del Rosario, Revista Ciencias de la Salud, enero-junio. Vol. 3, N.0 001, pp. 98 - 106, Bogotá, 2005.

REYES. Giovanna Liset, (compiladora), (2009), Diálogo de saberes: plantas medicinales, salud y cosmovisiones, Universidad Nacional de Colombia.

SCOTT, James (1998). Seeing a State: How Certain Shemes to Improve the Human Condition Have Failed. New Haven. Yale University Press.

VASCO, B. y HERNÁNDEZ, B. (2005), Agentes de Salud en algunas zonas del Cauca: Saberes Médicos al servicio de la comunidad, Universidad del Cauca, Licenciatura en Etnoeducación.

ZULUAGA, G. y CORREAL, C. (compiladores), (2002)Seminario Internacional de Etnomedicina: Aproximación al conocimiento de sistemas tradicionales de Salud, Universidad del Bosque, Instituto de Etnobiología, The Amazon Conservation Team, noviembre del 2002.

UNIVERSIDAD DEL ROSARIO, Revista medicina indígena y occidental: Diálogo de saberes, fascículo núm. 1. 